\title{
Finite Element Method and numerical study for a super-linear reaction-diffusion problem with integral conditions
}

Research Article

\author{
Benamira Sihem $^{1 *}$, Oussaeif Taki eddine ${ }^{2} \dagger \ddagger$, Dehilis Sofiane ${ }^{2} \S$, Bouziani Abdelfatah A $^{\natural}$ \\ 1 Département de mathematique, Université des fréres Mentouri Constantine 1, Algeria. \\ 2 Deapartment of Mathematics, University of Oum El Bouaghi, Algeria.
}

\begin{abstract}
In this work, we prove the existence, uniqueness, and continuous dependence of generalized solution of a nonlinear reaction-diffusion problem with only integral terms in the boundaries, by using the finite element method.Also we have developed an efficient numerical finite difference schemes. Some numerical results are reported to show the efficiency and accuracy of the scheme.

MSC: $\quad 35 \mathrm{~A} 05 ; 35 \mathrm{~A} 07 ; 35 \mathrm{~K} 50 ; 35 \mathrm{Q} 80$

Keywords: Non-local conditions • Integral conditions • finite element method • A priori estimates • super-linear parabolic equation $\bullet$ numerical study
\end{abstract}

\section{Introduction and notation}

In the recent years, a new attention has been given to reaction-diffusion problem which involve an integral over the spatial domain of a function of the desired solution on the boundary conditions; see [1 - 21]. The purpose of this paper is to prove the existence and uniqueness of a solution for the following non linear reaction diffusion problem with only integral conditions.

The plan of this paper is as follows. In section 2 we give some notations used through out the paper. Section 3 is devoted to statement of the problem. In section 4 we construct an approximate solution using finite element

* E-mail: s.benamira@yahoo.fr

$\dagger$ E-mail: takieddine.oussaeif@gmail.com

¥ E-mail: taki_maths@live.fr

$\S$ E-mail: dehilissofiane@yahoo.fr

ฯ E-mail: aefbouziani@yahoo.fr 
method. in section 5 we give some a priori estimates. Finally in the section 6 we prove the convergence and we give the existence result where we prove the uniqueness and the continuous dependence of solution.

Let $L^{2}(\Omega)$ be the usual space of square integrable functions ; its scalar product is denoted by (.,.) and its associated norm by $\|$.$\| . We denote by C_{0}(\Omega)$ the space of continuous functions with compact support in $\Omega$.

Definition 1.1.

We denote by $B_{2}^{m}(\Omega)$ called the Bouziani space, the Hilbert space defined of $C_{0}(\Omega)$ for the scalar product

$$
(z, w)_{B_{2}^{m}(\Omega)}=\int_{\Omega} \Im_{x}^{m} z \cdot \Im_{x}^{m} w d x
$$

where

$$
\Im_{x}^{m} z=\int_{\Omega} \frac{(x-\xi)^{m-1}}{(m-1) !} z(\xi) d \xi
$$

by the norm of the function $z$ from $B_{2}^{m}(\Omega)$, the nonnegative number

$$
\|z\|_{B_{2}^{m}(\Omega)}=\left(\int_{\Omega}\left(\Im_{x}^{m} z\right)^{2} d x\right)^{\frac{1}{2}}<\infty
$$

then the inequality

$$
\|z\|_{B_{2}^{m}(\Omega)}^{2} \leq \frac{(\beta-\alpha)^{2}}{2}\|z\|_{B_{2}^{m-1}(\Omega)}^{2}, m \geq 1,
$$

holds for every $z \in B_{2}^{m-1}(\Omega)$, and the embedding

$$
B_{2}^{m-1}(\Omega) \hookrightarrow B_{2}^{m}(\Omega)
$$

is continuous .

\section{Remark 1.1.}

If $m=0$, the space $B_{2}^{0}(\Omega)$ coincides with $L^{2}(\Omega)$.

\section{Definition 1.2.}

We denote by $L_{0}^{2}(\Omega)$ the space consisting of elements $z(x)$ of the space $L^{2}(\Omega)$ verifying

$$
\int_{\Omega} x^{k} z(x) d x=0(k=0,1)
$$

Let $\mathrm{X}$ be a space with a norm denoted by $\|\cdot\|_{X}$

Definition 1.3.

(i) Denote by $L^{2}(I, X)$ the set of all measurable abstract functions $u(., t)$ from $I$ into $X$ such that

$$
\|u\|_{L^{2}(I, X)}=\left(\int_{I}\|u(., t)\|_{x}^{2} d t\right)^{\frac{1}{2}}<\infty .
$$

(ii)Let $C(\bar{I} ; X)$ be the set of all continuous functions $u(., t): \bar{I} \longrightarrow X$ with

$$
\|u\|_{C(\bar{I} ; X)}=\max \|u(., t)\|_{X}<\infty
$$


Lemma 1.1.

Let be $v:[0, T] \rightarrow H$ be a Bochner integrable function and let $A \subset[0, T]$, any measurable subset, so: $i$ ) the function $\|v(.)\|_{H}:[0, T] \rightarrow H$ is Lebesgue integrable and we have,

$$
\left\|\int_{A} v(t) d t\right\|_{H} \leq \int_{A}\|v(t)\|_{H} d t
$$

ii) for each $\varphi \in H$, the function $(v(.), \varphi)_{H}:[0, T] \rightarrow \mathbb{R}$ is Lebesgue integrable and we have,

$$
\left(\int_{A} v(t) d t, \varphi\right)_{H}=\int_{A}(v(t), \varphi)_{H} d t
$$

Lemma 1.2.

Let $M$ be a linear closed subspace from a Hilbert space $H$. So for every $h \in H$, there exists a unique $u \in M$ such that:

$$
\|h-u\|_{H}=\min _{v \in M}\|h-v\|_{H},
$$

the element $u$ is called the orthogonal projection of $h$ on $M$ relatively to the inner product (.,.) and we note $u=P_{M} h$. Furthermore, we have the following Pythagorean relation

$$
\|h\|_{H}^{2}=\left\|P_{M} h\right\|_{H}^{2}+\left\|h-P_{M} h\right\|_{H}^{2} .
$$

Theorem 1.1 (Cauch- Schwarz inequality).

Let be $f$ and $g$ two functions of $L^{2}(\Omega)$; so

$$
f . g \in L^{1}(\Omega),
$$

and

$$
\int_{\Omega}|f \cdot g| \leq\|f\|_{L^{2}} \cdot\|g\|_{L^{2}} .
$$

Theorem 1.2 (The Cauchy inequality).

Let be $a, b \in \mathbb{R}$, and every $\varepsilon>0$, we have

$$
|a b| \leq \frac{\varepsilon}{2} a^{2}+\frac{1}{2 \varepsilon} b^{2} .
$$

Lemma 1.3 (Gronwall lemma).

Let $h(t)$ and $y(t)$ be two real integrable functions on the interval $I, h(\tau)$ nondeceasing, and c a positive constant if

$$
y(t) \leq h(t)+c \int_{0}^{t} y(\tau) d \tau \quad \forall t \in I,
$$

then

$$
y(t) \leq h(t) e^{c t} \quad \forall t \in I .
$$

\section{Definition 1.4.}

We call a nonlinear differential system the system of the form

$$
\dot{X}(t)=F[X(t)]
$$

$t$ is a real

where $f_{i}$ are continuous functions.

$$
X(t)=\left(\begin{array}{c}
x_{1}(t) \\
x_{2}(t) \\
\cdot \\
\cdot \\
\cdot \\
x_{n}(t)
\end{array}\right), \quad F(t)=\left(\begin{array}{c}
f_{1}(t) \\
f_{2}(t) \\
\cdot \\
\cdot \\
\cdot \\
f_{n}(t)
\end{array}\right)
$$


Definition 1.5.

Let be

$$
X(t): \begin{array}{ll}
I \subset \mathbb{R} & \longrightarrow \mathbb{R}^{n} \\
x & \longrightarrow x(t)
\end{array}
$$

$X$ is the solution of the system (11), if $X$ is derivable and continuous function, for every each $t \in I, X(t) \in I$ and $X(t)=F(X(t))$

Theorem 1.3 (The unicity of solution).

We suppose that $F$ is derivable continuous function on $E \subset \mathbb{R}^{n}$. So for every each initial condition for $t_{0} \in I$ and $X_{0} \in E$ the solution of the system (11) if it exists it is unique.

Theorem 1.4 (Local existence of solution).

Let be $t_{0} \in \mathbb{R}$ and $X_{0} \in \mathbb{R}^{n}$. If $F$ is derivable continuous on $X_{0}$, it exists $h>0$ such that the solution of the system (11) verifying $X\left(t_{0}\right)=X_{0}$ exists on the interval $\left[t_{0}, t_{0}+h\right]$.

Theorem 1.5 (Global existence of solution).

If $F$ is derivable continuous function on $\mathbb{R}^{n}$ and if the solution of the system (11) verifying $X(0)=X_{0}$ is bounded on the interval which it exists so the solution exists on $I=[0,+\infty]$.

See artical [18].

\section{Statement of the problem}

Let be the problem

$$
\frac{\partial u(x, t)}{\partial t}-\alpha \frac{\partial^{2} u(x, t)}{\partial x^{2}}-(u(x, t))^{p}=f(x, t)
$$

with the initial condition

$$
u(x, 0)=u^{0}
$$

and the boundary integral conditions

$$
\left\{\begin{array}{c}
\int_{0}^{1} u(x, t) d x=0 \\
\int_{0}^{1} x u(x, t) d x=0
\end{array},\right.
$$

with $t \in[0, T], T<\infty, \alpha \in \mathbb{R}_{+}^{*}, p \in \mathbb{N}^{*}, x \in[0,1]$.

Through the paper, we will make the following assumptions:

$\left(H_{1}\right): f \in L^{2}\left(0, T ; B_{2}^{1}(0,1)\right),\left(H_{2}\right): u^{0} \in V$ where $V$ is defined in the following way

$$
V=\left\{v \in L^{2}(0,1): \int_{0}^{1} v(x) d x=\int_{0}^{1} x v(x) d x=0\right\}
$$

Since $V$ is the null space of the continuous linear mapping

$$
g: L^{2}(0,1) \longrightarrow \mathbb{R}^{2}, \Phi \longrightarrow g(\Phi)=\left(\int_{0}^{1} \Phi(x) d x, \int_{0}^{1} x \Phi(x) d x\right)
$$


it is closed linear subspace of $L^{2}(0,1)$, consequently $V$ is a Hilbert space for (.,.). Moreover for a given function $w(x, t)$, the notation $w(t)$ is used for the same function considered as an abstract function of the variable $t .\left(H_{3}\right)$ : $f(t, w) \in L^{2}(0,1)$ for each $(t, w) \in I \times L^{2}(0,1)$ and the following Lipschitz condition

$$
\begin{aligned}
& \left\|f(t, w)-f\left(t^{\prime}, w^{\prime}\right)\right\|_{B_{2}^{1}(0,1)} \\
& \leq M\left[\left|t-t^{\prime}\right|\left(1+\|w\|_{B_{2}^{1}(0,1)}+\left\|w^{\prime}\right\|_{B_{2}^{1}(0,1)}\right)+\left\|w-w^{\prime}\right\|_{B_{2}^{1}(0,1)}\right] .
\end{aligned}
$$

Definition 2.1.

A weak solution of problem (13) - (15) means a function

$$
u:[0, T] \longrightarrow L^{2}(0,1)
$$

such that

(i) $u \in L^{2}\left(0, T ; B_{2}^{1}(0,1)\right)$,

(ii) $u$ has a strong derivative $\frac{d u}{d t} \in L^{2}\left(0, T ; B_{2}^{1}(0,1)\right)$, (iii) $u(0)=u^{0}$, (iv) The identity :

$$
\left(\frac{d u(t)}{\partial t}, v\right)_{B_{2}^{1}(0,1)}+\alpha(u(t), v)-\left(u^{p}(x, t), v\right)_{B_{2}^{1}(0,1)}=(f(x, t), v) .
$$

\section{Construction of an approximate solution}

Let $\varphi_{1}, \varphi_{2}, \ldots, \varphi_{N}, \ldots$ be a Hilbertian basis of $V$, such that we devise $[\theta, \beta]$ on $N+1$ parts $\left(N \in \mathbb{N}^{*}\right)$ and we pose

$$
h=\frac{1}{N+1}, t_{i}=i h, i=0,1,2, \ldots, N+1
$$

We define functions $\left(\varphi_{i}\right)$ by

$$
\varphi_{i}(x)= \begin{cases}\frac{x-x_{i-1}}{x_{i}-x_{i-1}}, & x_{i-1} \leq x \leq x_{i}, \\ \frac{x-x_{i}}{x_{i+1}-x_{i}}, & x_{i} \leq x \leq x_{i+1}, \\ 0, & \text { ailleurs }\end{cases}
$$

For every each functions $\left(\varphi_{i}\right)$ are of degree 1 with $\varphi_{i}\left(x_{j}\right)=\delta_{i j}$.

Let $\left(V_{n}\right)$ the subspace from $V$ generated by the first $n$ elements of the basis. We have to find for each $n \in \mathbb{N}^{*}$, the approximate solution which has the following form.

$$
u_{n}(x, t)=\sum_{i=1}^{n} g_{i n}(t) \varphi_{i}(x), \quad(x, t) \in(0,1) \times[0, T],
$$

where $g_{\text {in }} \in H^{1}(0, T)$ are unknown functions for the moment. As we have that $u^{0} \in V$ and $V_{n}$ is a closed subspace from $V$, we can define in a unique way $u_{n}^{0}$ by

$$
u_{n}^{0}=P_{V_{n}} u^{0}
$$


where $P_{V_{n}}$ is define in lemma (1). By the virtue of the density of $\cup V_{n}$ in $V$ it follows that

$$
u_{n}^{0} \longrightarrow u^{0} \text { in } V \text { if } n \longrightarrow \infty \text {. }
$$

We note by $\left(g_{i n}^{0}\right)$ the coordinates of $u_{n}^{0}$ in the basis $\left(\varphi_{i}\right)_{i=1}^{n}$ of $V_{n}$ that is

$$
u_{n}^{0}=\sum_{i=1}^{n} g_{i n}^{0} \varphi_{i}
$$

so, we have to find

$$
u_{n} \in H^{1}\left(0, T ; V_{n}\right)
$$

solution of the differential system

$$
\begin{gathered}
\left(\frac{d u_{n}}{d t}, \varphi_{j}\right)_{B_{2}^{1}(0,1)}+\alpha\left(u_{n}, \varphi_{j}\right)-\left(u_{n}^{p}, \varphi_{j}\right)_{B_{2}^{1}(0,1)}=\left(f(x, t), \varphi_{j}\right)_{B_{2}^{1}(0,1)} \\
u_{n}(0)=u_{n}^{0}
\end{gathered}
$$

By replacing $u_{n}$ by (17) and by using the following notations

$$
\begin{array}{ll}
\alpha_{i j}=\left(\varphi_{i}, \varphi_{j}\right)_{B_{2}^{1}(\Omega)} & , A=\left(\alpha_{i j}\right)_{1 \leq i, j \leq n}, \\
B_{i j}=\left(\varphi_{i}, \varphi_{j}\right) & , B=\left(B_{i j}\right)_{1 \leq i, j \leq n}, \\
C_{j}=\left(u_{n}^{p}, \varphi_{j}\right)_{B_{2}^{1}(0,1)} & , C=\left(C_{j}\right)_{1 \leq j \leq n,} \\
F_{j}(t)=\left(f, \varphi_{j}\right)_{B_{2}^{1}(0,1)}, & \overrightarrow{F(t)}=\left(F_{j}(t)\right)_{j=1}^{n},
\end{array}
$$

and

$$
\overrightarrow{g_{n}(t)}=\left(g_{i_{n}}(t)\right)_{i=1}^{n}, \overrightarrow{g_{n}^{0}}=\left(g_{i_{n}}^{0}\right)_{i=1}^{n}
$$

The system (22) can be written as follows

$$
A \frac{\overrightarrow{d g_{n}}}{d t}+\alpha B \overrightarrow{g_{n}}+C=\overrightarrow{F(t)}
$$

which is a nonlinear differential system.

We easily prove that $A$ is regular matrix, and by virtue definition (1.4), (1.5) and Theorems (1.3), (1.4) and (1.5), so the system (24) has a unique solution $\overrightarrow{g_{n}} \in\left[H^{1}(0, T)\right]^{n}$.

Lemma 3.1.

For every $n \geq 1$, the problem (22) - (23) has a unique solution $u_{n} \in H^{1}\left(0, T ; V_{n}\right)$ which has the form (17). 


\section{A-priori estimates for approximations}

Lemma 4.1.

For every $n \in \mathbb{N}^{*}$ functions $u_{n} \in H^{1}\left(0, T ; V_{n}\right)$ solutions of $(22)$ verify

$$
\int_{0}^{t}\left\|u_{n}\right\|^{2} d \tau \leq \frac{K}{2 \alpha-1-\frac{p}{2}}
$$

and

$$
\int_{0}^{t}\left\|\frac{d u_{n}}{d t}\right\|_{B_{2}^{1}(0,1)}^{2} d \tau \leq L
$$

where $K$ and $L$ are two positive constants such that,

$$
\alpha>\frac{1}{2}+p
$$

Proof. Multiplying the integral identity (22) by $g_{j n}(t)$ and summing up for $j=1, \ldots, n$ and integrating the resulting over $(0, t)$, we obtain

$$
\begin{aligned}
& \frac{1}{2}\left\|u_{n}\right\|_{B_{2}^{1}(0,1)}^{2}+\alpha \int_{0}^{t}\left\|u_{n}\right\|^{2} d \tau \\
& =\int_{0}^{t}\left(f, u_{n}\right)_{B_{2}^{1}(0,1)} d \tau+\int_{0}^{t}\left(u_{n}^{p}, u_{n}\right)_{B_{2}^{1}(0,1)} d \tau+\frac{1}{2}\left\|u_{n}^{0}\right\|_{B_{2}^{1}(0,1)}^{2} .
\end{aligned}
$$

We have

$$
\left\|u_{n}^{0}\right\|_{B_{2}^{1}(0,1)}^{2} \leq\left\|u^{0}\right\|_{B_{2}^{1}(0,1)}^{2} \leq \frac{1}{2}\left\|u^{0}\right\|^{2},
$$

so

$$
\begin{aligned}
& \left\|u_{n}\right\|_{B_{2}^{1}(0,1)}^{2}+2 \alpha \int_{0}^{t}\left\|u_{n}\right\|^{2} d \tau \\
& =2 \int_{0}^{t}\left(f, u_{n}\right)_{B_{2}^{1}(0,1)} d \tau+2 \int_{0}^{t}\left(u_{n}^{p}, u_{n}\right)_{B_{2}^{1}(0,1)} d \tau+\frac{1}{4}\left\|u^{0}\right\|^{2},
\end{aligned}
$$

hence, thanks to the Cauchy inequality (29)

$$
\begin{aligned}
& \left\|u_{n}\right\|_{B_{2}^{1}(0,1)}^{2}+2 \alpha \int_{0}^{t}\left\|u_{n}\right\|^{2} d \tau \\
& \leq \int_{0}^{t}\|f\|_{B_{2}^{1}(0,1)}^{2} d \tau+\int_{0}^{t}\left\|u_{n}\right\|_{B_{2}^{1}(0,1)}^{2} d \tau+\int_{0}^{t}\left\|u_{n}^{p}\right\|_{B_{2}^{1}(0,1)}^{2} d \tau \\
& +\int_{0}^{t}\left\|u_{n}\right\|_{B_{2}^{1}(0,1)}^{2} d \tau+\frac{1}{4}\left\|u^{0}\right\|^{2},
\end{aligned}
$$

but we have

$$
\left\|u_{n}\right\|_{B_{2}^{1}(0,1)}^{2} \leq \frac{1}{2}\left\|u_{n}\right\|^{2},
$$

we get

$$
\begin{aligned}
& \left\|u_{n}\right\|_{B_{2}^{1}(0,1)}^{2}+(2 \alpha-1) \int_{0}^{t}\left\|u_{n}\right\|^{2} d \tau \\
& \leq \int_{0}^{t}\|f\|_{B_{2}^{1}(0,1)}^{2} d \tau+\frac{1}{4}\left\|u^{0}\right\|^{2}+\int_{0}^{t}\left\|u_{n}^{p}\right\|_{B_{2}^{1}(0,1)}^{2} d \tau,
\end{aligned}
$$


we have that

$$
\begin{aligned}
\int_{0}^{t}\left\|u_{n}^{p}\right\|_{B_{2}^{1}(0,1)}^{2} d \tau & =\int_{0}^{t}\left\|u_{n}^{p-1} \cdot u_{n}\right\|_{B_{2}^{1}(0,1)}^{2} d \tau \\
& \leq \frac{1}{2} \int_{0}^{t}\left\|u_{n}^{p-1}\right\|_{B_{2}^{1}(0,1)}^{2} d \tau+\frac{1}{2} \int_{0}^{t}\left\|u_{n}\right\|_{B_{2}^{1}(0,1)}^{2} d \tau \\
& \leq \frac{1}{2} \int_{0}^{t}\left\|u_{n}^{p-1}\right\|_{B_{2}^{1}(0,1)}^{2} d \tau+\frac{1}{4} \int_{0}^{t}\left\|u_{n}\right\|^{2} d \tau,
\end{aligned}
$$

substituting (32) in (31) we have

$$
\begin{aligned}
& \left\|u_{n}\right\|_{B_{2}^{1}(0,1)}^{2}+\left(2 \alpha-\frac{5}{4}\right) \int_{0}^{t}\left\|u_{n}\right\|^{2} d \tau \\
& \leq \int_{0}^{t}\|f\|_{B_{2}^{1}(0,1)}^{2} d \tau+\frac{1}{4}\left\|u^{0}\right\|^{2}+\int_{0}^{t}\left\|u_{n}^{p-1}\right\|_{B_{2}^{1}(0,1)}^{2} d \tau .
\end{aligned}
$$

But

$$
\begin{aligned}
\int_{0}^{t}\left\|u_{n}^{p-1}\right\|_{B_{2}^{1}(0,1)}^{2} d \tau & =\int_{0}^{t}\left\|u_{n}^{p-2} \cdot u_{n}\right\|_{B_{2}^{1}(0,1)}^{2} d \tau \\
& \leq \frac{1}{2} \int_{0}^{t}\left\|u_{n}^{p-2}\right\|_{B_{2}^{1}(0,1)}^{2} d \tau+\frac{1}{2} \int_{0}^{t}\left\|u_{n}\right\|_{B_{2}^{1}(0,1)}^{2} d \tau \\
& \leq \frac{1}{2} \int_{0}^{t}\left\|u_{n}^{p-2}\right\|_{B_{2}^{1}(0,1)}^{2} d \tau+\frac{1}{4} \int_{0}^{t}\left\|u_{n}\right\|^{2} d \tau .
\end{aligned}
$$

Since (34) so (33) can be written

$$
\begin{aligned}
& \left\|u_{n}\right\|_{B_{2}^{1}(0,1)}^{2}+\left(2 \alpha-1-\frac{1}{2}-\frac{1}{2}\right) \int_{0}^{t}\left\|u_{n}\right\|^{2} d \tau \\
& \leq \int_{0}^{t}\|f\|_{B_{2}^{1}(0,1)}^{2} d \tau+\frac{1}{2}\left\|u^{0}\right\|^{2}+\int_{0}^{t}\left\|u_{n}^{p-2}\right\|_{B_{2}^{1}(0,1)}^{2} d \tau
\end{aligned}
$$

after $p$ iteration we get

$$
\begin{aligned}
& \left\|u_{n}\right\|_{B_{2}^{1}(0,1)}^{2}+\left(2 \alpha-1-\frac{p}{2}\right) \int_{0}^{t}\left\|u_{n}\right\|^{2} d \tau \\
& \leq \int_{0}^{t}\|f\|_{B_{2}^{1}(0,1)}^{2} d \tau+\frac{1}{4}\left\|u^{0}\right\|^{2}+\int_{0}^{t}\left\|\left(u_{n}\right)^{0}\right\|_{B_{2}^{1}(0,1)}^{2} d \tau
\end{aligned}
$$

so

$$
\begin{aligned}
& \left\|u_{n}\right\|_{B_{2}^{1}(0,1)}^{2}+\left(2 \alpha-1-\frac{p}{2}\right) \int_{0}^{t}\left\|u_{n}\right\|^{2} d \tau \\
& \leq \int_{0}^{t}\|f\|_{B_{2}^{1}(0,1)}^{2} d \tau+\frac{1}{4}\left\|u^{0}\right\|^{2}+\frac{T}{2}
\end{aligned}
$$

Let be

$$
K=\int_{0}^{t}\|f\|_{B_{2}^{1}(0,1)}^{2} d \tau+\frac{1}{4}\left\|u^{0}\right\|^{2}+\frac{T}{2}
$$

we get

$$
\left\|u_{n}\right\|_{B_{2}^{1}(0,1)}^{2} \leq K
$$

and

$$
\int_{0}^{t}\left\|u_{n}\right\|^{2} d \tau \leq \frac{K}{2 \alpha-1-\frac{p}{2}}
$$

on the other hand multiplying (22) by $\frac{d g_{j n}}{d t}$ and sum up for $j=1, \ldots, n$ we obtain

$$
\left\|\frac{d u_{n}}{d t}\right\|_{B_{2}^{1}(0,1)}^{2}+\frac{\alpha}{2} \frac{d}{d t}\left\|u_{n}\right\|^{2}=\left(f, \frac{d u_{n}}{d t}\right)_{B_{2}^{1}(0,1)}+\left(u_{n}^{p}, \frac{d u_{n}}{d t}\right)_{B_{2}^{1}(0,1)}
$$


integrating (41) over $(0, t)$

$$
\begin{aligned}
& 2 \int_{0}^{t}\left\|\frac{d u_{n}}{d t}\right\|_{B_{2}^{1}(0,1)}^{2} d \tau+\alpha\left\|u_{n}\right\|^{2} \\
& =2 \int_{0}^{t}\left(f, \frac{d u_{n}}{d t}\right)_{B_{2}^{\frac{1}{2}(0,1)}} d \tau+2 \int_{0}^{t}\left(u_{n}^{p}, \frac{d u_{n}}{d t}\right)_{B_{2}^{1}(0,1)} d \tau+\alpha\left\|u^{0}\right\|^{2}
\end{aligned}
$$

applying the Cauchy inequality, we get

$$
\begin{aligned}
& 2 \int_{0}^{t}\left\|\frac{d u_{n}}{d t}\right\|_{B_{2}^{1}(0,1)}^{2} d \tau+\alpha\left\|u_{n}\right\|^{2} \\
& =2 \int_{0}^{t}\left(f, \frac{d u_{n}}{d t}\right)_{B_{2}^{1}(0,1)} d \tau+2 \int_{0}^{t}\left(u_{n}^{p}, \frac{d u_{n}}{d t}\right)_{B_{2}^{1}(0,1)} d \tau+\alpha\left\|u^{0}\right\|^{2}
\end{aligned}
$$

so

$$
\begin{aligned}
& \int_{0}^{t}\left\|\frac{d u_{n}}{d t}\right\|_{B_{2}^{1}(0,1)}^{2} d \tau+\alpha\left\|u_{n}\right\|^{2} \\
& \leq \int_{0}^{t}\|f\|_{B_{2}^{1}(0,1)}^{2} d \tau+\alpha\left\|u^{0}\right\|^{2}+\int_{0}^{t}\left\|u_{n}^{p}\right\|_{B_{2}^{1}(0,1)}^{2} d \tau
\end{aligned}
$$

but we have

$$
\begin{aligned}
\int_{0}^{t}\left\|u_{n}^{p}\right\|_{B_{2}^{1}(0,1)}^{2} d \tau & =\int_{0}^{t}\left\|u_{n}^{p-1} \cdot u_{n}\right\|_{B_{2}^{1}(0,1)}^{2} d \tau \\
& \leq \frac{1}{2} \int_{0}^{t}\left\|u_{n}^{p-1}\right\|_{B_{2}^{1}(0,1)}^{2} d \tau+\frac{1}{2} \int_{0}^{t}\left\|u_{n}\right\|_{B_{2}^{1}(0,1)}^{2} d \tau \\
& \leq \frac{1}{2} \int_{0}^{t}\left\|u_{n}^{p-1}\right\|_{B_{2}^{1}(0,1)}^{2} d \tau+\frac{1}{2} K T \quad \text { see equation (39) } \\
& \leq \frac{1}{2} \int_{0}^{t}\left\|u_{n}^{p-2} \cdot u_{n}\right\|_{B_{2}^{1}(0,1)}^{2} d \tau+\frac{1}{2} K T \\
& \leq \frac{1}{2}\left[\frac{1}{2} \int_{0}^{t}\left\|u_{n}^{p-2}\right\|_{B_{2}^{1}(0,1)}^{2} d \tau+\frac{1}{2} \int_{0}^{t}\left\|u_{n}\right\|_{B_{2}^{1}(0,1)}^{2} d \tau\right]+\frac{1}{2} K T \\
& \leq \frac{1}{2} \cdot \frac{1}{2} \int_{0}^{t}\left\|u_{n}^{p-2}\right\|_{B_{2}^{1}(0,1)}^{2} d \tau+\frac{1}{2} \cdot \frac{1}{2} \cdot K T+\frac{1}{2} K T
\end{aligned}
$$

after $p$ iteration we get

$$
\int_{0}^{t}\left\|u_{n}^{p}\right\|_{B_{2}^{\frac{1}{2}(0,1)}}^{2} d \tau \leq T\left(\frac{1}{2^{p+1}}\left\|u_{0}\right\|^{2}+K\left(\frac{1}{2^{p}}+\frac{1}{2}\right)\right),
$$

substituting (45) in (44) we get

$$
\begin{aligned}
& \int_{0}^{t}\left\|\frac{d u_{n}}{d t}\right\|_{B_{2}^{1}(0,1)}^{2} d \tau+\alpha\left\|u_{n}\right\|^{2} \\
& \leq \int_{0}^{t}\|f\|_{B_{2}^{1}(0,1)}^{2} d \tau+\alpha\left\|u^{0}\right\|^{2}+T\left(\frac{1}{2^{p+1}}\left\|(u)^{0}\right\|^{2}+K\left(\frac{1}{2^{p}}+\frac{1}{2}\right)\right) .
\end{aligned}
$$

Let be

$$
L=\int_{0}^{t}\|f\|_{B_{2}^{1}(0,1)}^{2} d \tau+\alpha\left\|u^{0}\right\|^{2}+T\left(\frac{1}{2^{p+1}}+K\left(\frac{1}{2^{p}}+\frac{1}{2}\right)\right),
$$

so we have

$$
\int_{0}^{t}\left\|\frac{d u_{n}}{d t}\right\|_{B_{2}^{1}(0,1)}^{2} d \tau \leq L
$$




\section{Convergence and existence result}

Theorem 5.1.

There exist a function $u \in L^{2}(0, T ; V)$ with

$$
\frac{d u}{d t} \in L^{2}\left(0, T ; B_{2}^{1}(0,1)\right)
$$

and a subsequence $\left(u_{n_{k}}\right)_{k} \subseteq\left(u_{n}\right)_{n}$ such that

$$
u_{n_{k}} \rightarrow u \text { in } L^{2}(0, T ; V)
$$

and

$$
\frac{d u_{n_{k}}}{d t} \rightarrow \frac{d u}{d t} \text { in } L^{2}\left(0, T ; B_{2}^{1}(0,1)\right),
$$

when $n \longrightarrow \infty$.

Proof. See article [3]

Theorem 5.2.

The limit function u from Theorem (5.1) is the unique weak solution to problem (13) - (15) in the sense of definition (2.1).

Proof. One : Existence. We have to show that the limit function $u$ satisfies all conditions $(i)-(i v)$ of definition (2.1) . Obviously, in light of properties of function $u$ the first two conditions are already seen. On the other hand, from $u(t)=u^{0}+\int_{0}^{t} \Psi(s) d s, t \in[0, T]$, written in the proof of Theorem (5.1), we have directly $u(0)=u^{0}$, so the initial condition is also fulfilled, now we have to see that integral identity obeyed by $u$, for this, writing (22) for $n=n_{k}$ and integrating on $[0, t]$, it comes

$$
\begin{aligned}
& \int_{0}^{t}\left(\frac{\partial u_{n_{k}}(s)}{\partial s}, \varphi_{j}\right)_{B_{2}^{1}(0,1)} d s+\alpha \int_{0}^{t}\left(u_{n_{k}}(s), \varphi_{j}\right) d s \\
& -\int_{0}^{t}\left(u_{n_{k}}^{p}(s), \varphi_{j}\right)_{B_{2}^{1}(0,1)} d s \\
& =\int_{0}^{t}\left(f(x, s), \varphi_{j}\right)_{B_{2}^{1}(0,1)} d s ; \quad \forall t \in[0, T], \quad j=1, \ldots, n_{k} .
\end{aligned}
$$

By performing a limit process $k \longrightarrow \infty$ in (51), we get owing (49) and (50)

$$
\begin{aligned}
& \int_{0}^{t}\left(\frac{\partial u(s)}{\partial s}, \varphi_{j}\right)_{B_{2}^{1}(0,1)} d s+\alpha \int_{0}^{t}\left(u(s), \varphi_{j}\right) d s-\int_{0}^{t}\left(u^{P}(s), \varphi_{j}\right)_{B_{2}^{1}(0,1)} d s \\
& =\int_{0}^{t}\left(f(x, s), \varphi_{j}\right)_{B_{2}^{1}(0,1)} d s ; \quad \forall t \in[0, T], \quad j=1, \ldots, n_{k} .
\end{aligned}
$$

Differentiating this latter with respect to $t$ we get

$$
\begin{aligned}
& \left(\frac{\partial u(t)}{\partial t}, \varphi_{j}\right)_{B_{2}^{1}(0,1)}+\alpha\left(u(t), \varphi_{j}\right) \\
& -\left(u^{p}(t), \varphi_{j}\right)_{B_{2}^{1}(0,1)} \\
& =\left(f(x, t), \varphi_{j}\right)_{B_{2}^{1}(0,1)} \quad \forall t \in[0, T], j \geq 1 .
\end{aligned}
$$


From where $(i v)$ is obtained due the density of $\left(\cup_{n} V_{n}\right)$ in $V$. Thus, $u$ weakly solves problem (13) - (14) . Two : Uniqueness . Writing the problem (13) - (15) in the form

$$
\frac{\partial u(x, t)}{\partial t}-\alpha \frac{\partial^{2} u(x, t)}{\partial t^{2}}=f(x, t, u(x, t))
$$

which

$$
f(x, t, u(x, t))=(u(x, t))^{p}+f(x, t) .
$$

Let us $(\tilde{u}, \stackrel{\circ}{u})$ two weak solutions of $(54)$ we get

$$
\left(\frac{d \tilde{u}(t)}{\partial t}, v\right)_{B_{2}^{1}(0,1)}+\alpha(\tilde{u}(t), v)=(f(\tilde{u}, x, t), v)_{B_{2}^{1}(0,1)}
$$

and

$$
\left(\frac{d \stackrel{\circ}{u}(t)}{\partial t}, v\right)_{B_{2}^{1}(0,1)}+\alpha(\stackrel{\circ}{u}(t), v)=(f(\stackrel{\circ}{u}, x, t), v)_{B_{2}^{1}(0,1)},
$$

subtructing the identity (57) from (56) we get for $v=\stackrel{u}{-} \tilde{u}$

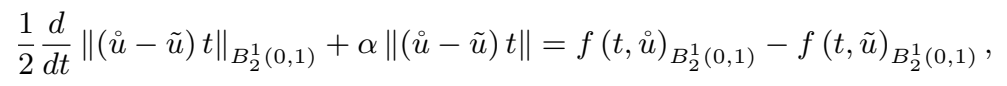

integrating (58) and putting $u(t)=\stackrel{\leftrightarrow}{u}-\tilde{u}$ we have

$$
\begin{aligned}
\|u(t)\|_{B_{2}^{1}(0,1)}^{2}+2 \alpha \int_{0}^{t}\|u(\tau)\|^{2} d \tau & =2 \int_{0}^{t}(f(\tau, \stackrel{\leftrightarrow}{u})-f(\tau, \tilde{u}), u)_{B_{2}^{1}(0,1)} d \tau, \\
& \leq 2 \int_{0}^{t} \| f\left(\tau, \stackrel{u}{u}-f(\tau, \tilde{u})\|\cdot\| u(\tau) \|_{B_{2}^{1}(0,1)} d \tau\right. \\
& \leq 2 M \int_{0}^{t}\|u(\tau)\|_{B_{2}^{1}(0,1)}^{2} d \tau .
\end{aligned}
$$

From where Gronwalls lemma yields $\|u(\tau)\|_{B_{2}^{1}(0,1)}^{2}=0 \Longrightarrow \stackrel{\circ}{=} \tilde{u}$; So, we have the uniqueness of the solution.

\section{Proposition 5.1.}

The sequence $\left(u_{n}\right)_{n}$ totally converges to $u$ in $L^{2}(0, T ; V)$.

Proof. The key point is to reason by absurdity, so we suppose that $\left(u_{n}\right)$ is not converging to $u$ in $L^{2}(0, T ; V)$ then

$$
\begin{gathered}
\exists \varepsilon \geq 0, \exists v \in L^{2}(0, T ; V), \exists\left(u_{\xi}\right)_{\xi} \subset\left(u_{n}\right)_{n}: \\
\left|\int_{0}^{T}\left(u_{\xi}(t)-u(t), v(t)\right) d t\right| \geq \varepsilon, \forall v,
\end{gathered}
$$

but $\left(u_{\xi}\right)_{\xi}$ is bounded in $L^{2}(0, T ; V)$, consequently we can construct a subsequence $\left(u_{\xi_{j}}\right)$ which weakly converges in $L^{2}(0, T ; V)$ towards a certain element $w \in L^{2}(0, T ; V)$, and while reasoning exactly as for the function $u$ from the theorem (5.1), we prove that $u$ is another solution for the problem (13) - (15), which implies,taking into account uniqueness in the problem in question, that $w$ is none other than $u$, so

$$
\lim _{\xi \longrightarrow \infty} \int_{0}^{T}\left(u_{\xi}(t)-u(t), v(t)\right) d t=0
$$


which is in contradiction with (60), thus

$$
u_{n} \rightarrow u \text { in } L^{2}(0, T ; V)
$$

Theorem 5.3.

Let be $u^{0}, u_{*}^{0} \in V, f, f_{*} \in L^{2}\left(O, T ; B_{2}^{1}(0,1)\right)$, and let $u$ and $u_{*}$ be the corresponding weak solutions satisfying assumptions $\left(H_{1}\right)-\left(H_{3}\right)$, if the following inequality

$$
\left\|f(t, v)-f_{*}(t, w)\right\|_{B_{2}^{1}(0,1)} \leq a(t)+b\|v-w\|_{B_{2}^{1}(0,1)}, \quad \forall t \in I, \forall v, w \in V
$$

holds for some continuous nonnegative $a(t) \in I$ and some constant $b \geq 0$ we have the estimate

$$
\left\|u-u_{*}\right\|_{B_{2}^{1}(0,1)}^{2} \leq\left(\left\|u^{0}-u_{*}^{0}\right\|_{B_{2}^{1}(0,1)}^{2}+\int_{0}^{t} a^{2}(\tau) d \tau\right) e^{(2 b+1) t}
$$

Proof. We take the difference identities (56) - (57) corresponding to $u, u_{*}$ and $f, f_{*}$

$$
\begin{gathered}
\left\|u-u_{*}\right\|_{B_{2}^{1}(0,1)}^{2}+2 \alpha \int_{0}^{t}\left\|u(\tau)-u_{*}(\tau)\right\|^{2} d \tau \\
\leq\left\|u^{0}-u_{*}^{0}\right\|_{B_{2}^{1}(0,1)}^{2} \\
+2 \int\left\|f(\tau, u)-f_{*}\left(\tau, u_{*}\right)\right\|_{B_{2}^{1}(0,1)} \cdot\left\|u(\tau)-u_{*}(\tau)\right\|_{B_{2}^{1}(0,1)} d \tau,
\end{gathered}
$$

applying the elementary algebraic inequality

$$
2 \alpha \beta \leq \alpha^{2}+\beta^{2} ; \quad \forall \alpha, \beta \in \mathbb{R}
$$

to the second term in the right hand side, we derive

$$
\begin{gathered}
\left\|u-u_{*}\right\|_{B_{2}^{1}(0,1)}^{2}+2 \alpha \int_{0}^{t}\left\|u(\tau)-u_{*}(\tau)\right\|^{2} d \tau \\
\leq\left\|u^{0}-u_{*}^{0}\right\|_{B_{2}^{1}(0,1)}^{2} \\
+\int_{0}^{t} a^{2}(\tau) d \tau+(2 b+1) \int_{0}^{1}\left\|u(\tau)-u_{*}(\tau)\right\|_{B_{2}^{1}(0,1)}^{2} d \tau
\end{gathered}
$$

from which the estimate (62) follows by means of Gromwell's lemma.

\section{Numerical study with finite difference schemes}

For the numerical solution of the considered problem (1.1)-(1.4) we apply the finite difference technique. First, we take a positive integers $N$ and $M$. We divide the intervals $[0,1]$ and $[0, T]$ into $M$ and $N$ subintervals of equal lengths $h=1 / M$ and $k=T / N$, respectively. By $u_{i}^{n}$, we denote the approximation to $u$ at the $i^{t h}$ grid-point and $n^{t h}$ time step. The Grid point $\left(x_{i}, t_{n}\right)$ are given by $x_{i}=i h, i=0,1,2, \ldots, M, t_{n}=n k, n=0,1,2, \ldots, N$. The notations $u_{i}^{n}$ and $f_{i}^{n}$, are used for the finite difference approximations of $u\left(x_{i}, t_{n}\right), f\left(x_{i}, t_{n}\right)$ respectively. 


\subsection{The forward time centred space (FTCS)}

We can approximate the time derivative by the forward difference quotient,and use the centred second-order approximation for the spatial derivative of second order in (3.1) to obtain :

$$
\frac{u_{i}^{n+1}-u_{i}^{n}}{k}=\alpha\left(\frac{u_{i-1}^{n}-2 u_{i}^{n}+u_{i+1}^{n}}{h^{2}}\right)+\left(u_{i}^{n}\right)^{p}+f_{i}^{n} .
$$

This scheme can be written as:

$$
u_{i}^{n+1}=r u_{i-1}^{n}+(1-2 r) u_{i}^{n}+r u_{i+1}^{n}+k\left(\left(u_{i}^{n}\right)^{p}+f_{i}^{n}\right)
$$

for $i=1,2, \ldots, M-1, n=0,1, \ldots, N$, and $r=\alpha k / h^{2}$.

This procedure is explicit and and we do not need to solve nonlinear algebraic equations. Order of accuracy of the scheme is $O(k)+O\left(h^{2}\right)$. We still have to determinates two unknowns $u_{0}^{n+1}$ and $u_{M+1}^{n+1}$, for this we approximate integrals in (2.3) numerically by trapezoidal rule ( We have chosen this approximation since it is of the same, second, order of accuracy in space as the methods used for the interior part of the problem ):

$$
\begin{gathered}
\int_{0}^{1} u\left(x, t^{n+1}\right) d x=\frac{h}{2}\left(u_{0}^{n+1}+2 \sum_{i=1}^{M-1} u_{i}^{n+1}+u_{M}^{n+1}\right)=0 \\
\int_{0}^{1} x u\left(x, t^{n+1}\right) d x=\frac{h}{2}\left(x_{0} u_{0}^{n+1}+2 \sum_{i=1}^{M-1} x_{i} u_{i}^{n+1}+x_{M} u_{M}\right)=0 .
\end{gathered}
$$

Thus, we can write

$$
\begin{gathered}
u_{0}^{n+1}+u_{M}^{n+1}=-2 \sum_{i=1}^{M-1} u_{i}^{n+1} \\
x_{0} u_{0}^{n+1}+x_{M} u_{M}^{n+1}=-2 \sum_{i=1}^{M-1} x_{i} u_{i}^{n+1}
\end{gathered}
$$

Hence we have:

$$
\begin{aligned}
& u_{0}^{n+1}=\frac{x_{M} z_{1}-z_{2}}{Y}, \\
& u_{M}^{n+1}=\frac{z_{2}-x_{0} z_{1}}{Y}
\end{aligned}
$$

where

$$
\begin{aligned}
& z_{1}=-2 \sum_{i=1}^{M-1} u_{i}^{n+1} \\
& z_{2}=-2 \sum_{i=1}^{M-1} x_{i} u_{i}^{n+1}
\end{aligned}
$$

and

$$
Y=x_{M}-x_{0} \neq 0
$$




\subsection{Numerical experiments}

To test the above algorithm we use example with known analytical solution as follows :

\section{Example 6.1.}

We consider the following problem

$$
\frac{\partial u}{\partial t}-\frac{\partial^{2} u}{\partial x^{2}}-u^{3}=f(x, t), \quad 0<x<1, \quad 0<t \leq T
$$

subject to the initial condition

$$
u(x, 0)=\cos (2 \pi x), \quad 0 \leq x<1,
$$

and the boundary integral conditions

$$
\begin{array}{ll}
\int_{0}^{1} u(x, t) d x=0, & 0<t \leq T, \\
\int_{0}^{1} x u(x, t) d x=0, & 0<t \leq T,
\end{array}
$$

Where

$$
f(x, t)=\cos (2 \pi x)\left(\sin (t)+4 \pi^{2} \cos (t)-\cos ^{3}(t) \cos ^{2}(2 \pi x)\right.
$$

Then the exact solution of the problem (6.1)-(6.4) is

$$
u(x, t)=\cos (2 \pi x) \cos (t) .
$$

In Table 1 and Table 2 we present results with $h=0.05,0.005$ using the FTCS scheme for $x=0.1$ and $t=$ $0.01,0.02,0.03, \ldots, 0.1$.

\begin{tabular}{|c|c|c|}
\hline$t_{i}$ & FTCS & exact \\
\hline 0.01 & 0.81118183 & 0.80897654 \\
0.02 & 0.81253938 & 0.80885520 \\
0.03 & 0.81332861 & 0.80865296 \\
$\ldots$ & $\ldots$ & $\ldots$ \\
0.1 & 0.81188905 & 0.80497528 \\
\hline
\end{tabular}

Table 1. Some numerical results at $x=0.1$ with $h=0.05$ and $r=0.4$

\begin{tabular}{|c|c|c|}
\hline$t_{i}$ & FTCS & exact \\
\hline 0.01 & 0.80899850 & 0.80897654 \\
0.02 & 0.80889228 & 0.80885520 \\
0.03 & 0.80870046 & 0.80865296 \\
$\ldots$ & $\ldots$ & $\ldots$ \\
0.1 & 0.80504396 & 0.80497528 \\
\hline
\end{tabular}

Table 2. Some numerical results at $x=0.1$ with $h=0.005$ and $r=0.4$ 


\section{References}

[1] A.Bouziani, On a class of nonlinear reaction-Diffusion systems with nonlocal boundary conditions, A analysis2004, 9(2004), 793-813.

[2] A.Bouziani, N.Merazga, S.Benamira, Galerkin method applied to a parabolic evolution problem with nonlocal boundary conditions. Non linear Analysis 69(2008), 1515-1524

[3] A.Bouziani, Mixed problem with boundary integral conditions for a certain parabolic equation, J. Appl. Math. Stochatic.Anal.9(1996), 323-330.

[4] A. Bouziani, N.Merazga, On a time-discretisation method for a semilinear heat equation with purely integral condition in a nonclassical function . Non linear analysis 66( 2007), 604-623.

[5] M.Dehghan, Fully explicit finite-differenc emethod for two-dimensional diffusion with an integral condition, nonlinear Anal. 48(2002), no. 5, 637-650.

[6] C.V.Pao, Numerical solutions of reaction-diffusion equations with nonlocal boundary conditions, J.Comput.Appl.Math. 136(2001), no.1-2, 227-243.

[7] T. E Oussaeif, A Bouziani, Solvability of nonlinear viscosity Equation with a boundary integral condition, J. Nonlinear Evol. Equ. Appl 3(2015), 31-45.

[8] T. E Oussaeif, A Bouziani; Solvability of Nonlinear Goursat Type Problem for Hyperbolic Equation with Integral Condition; Khayyam Journal of Mathematics 4, no.2, 198-213.

[9] Bouziani A, T-E Oussaeif and L. Benaoua, "A Mixed Problem with an Integral Two-Space-Variables Condition for Parabolic Equation with The Bessel Operator", Journal of Mathematics, 2013 (2013), Article ID 457631, 8 pages.

[10] Dhelis S, Bouziani A, Oussaeif T-E, Study of Solution for a Parabolic Integrodifferential Equation with the Second Kind Integral Condition, Int. J. Anal. Appl. 16 (2018), no.4, 569-593.

[11] O. Taki-Eddine, B Abdelfatah; A priori estimates for weak solution for a time-fractional nonlinear reactiondiffusion equations with an integral condition,Chaos, Solitons \& Fractals, 103 (2017), 79-89

[12] Oussaeif T-E, Bouziani A. Existence and uniqueness of solutions to parabolic fractional differential equations with integral conditions. Electron J. Differ. Equ. 179 (2014), 1-10.

[13] T-E Oussaeif, A Bouziani, Mixed Problem with an Integral Two-Space-Variables Condition for a Class of Hyperbolic Equations, International Journal of Analysis. 2013 (2013), 8 pages.

[14] T-E Oussaeif, A Bouziani, Mixed Problem with an Integral Two-Space-Variables Condition for a Parabolic Equation. International Journal of Evolution Equations, 9, no.2, 181-198.

[15] T.-E. Oussaeif, A. Bouziani, Mixed Problem with an Integral Two-Space-Variables condition for a Third Order Parabolic Equation, International Journal of Analysis and Applications, 12 (2016), no.2, 98-117.

[16] T.-E. Oussaeif, A. Bouziani, Solvability of nonlinear viscosity equation with a boundary integral condition, J. Nonl. Evol. Equ. Appl. 2015 (2015), no.3, 31-45. 
[17] T-E Oussaeif, A Bouziani and N Gattal, Numerical Solution of Mixed Problem of Parabolic Equation with an Integral Conditions by using Finite Difference and Orthogonal Function Approximation, Mathematica Moravica, 16 (2012), no.2, 89-98.

[18] Françoise Truc Systemes differentiels non lineaires October2010.

[19] T.-E. Oussaeif, A. Bouziani, Nonlocal problem for a third order partial differential equation of mixed type with an integral two-space-variables condition, Communications in Optimization Theory; 2017 (2017), 1-16, Article ID 16

[20] B. Sihem, O. Taki Eddine and B. Abdelfatah; Galerkin Finite Element Method for a Semi-linear Parabolic Equation with Purely Integral Conditions; Bol. Soc. Paran. Mat; DOI: 10.5269/bspm.44918

[21] B. Sihem, O. Taki Eddine and B. Abdelfatah, study a semi-linear pseudo-parabolic problem with Neumann and integral conditions by using Galerkin mixed finite element method; Communications in Nonlinear Analysis 9, Issue 1, 1-20. 